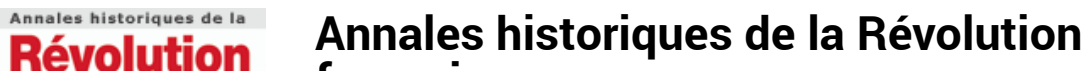

française française

347 | janvier-mars 2007

Varia

\section{Mœurs, vertu et corruption : Sade et le républicanisme classique}

\section{Martin Nadeau}

\section{(2) OpenEdition \\ 9 Journals}

Édition électronique

URL : https://journals.openedition.org/ahrf/8393

DOI : 10.4000/ahrf.8393

ISSN : 1952-403X

Éditeur :

Armand Colin, Société des études robespierristes

\section{Édition imprimée}

Date de publication : 1 mars 2007

Pagination : 29-46

ISSN : 0003-4436

\section{Référence électronique}

Martin Nadeau, « Mœurs, vertu et corruption : Sade et le républicanisme classique », Annales

historiques de la Révolution française [En ligne], 347 | janvier-mars 2007, mis en ligne le 01 mars 2010,

consulté le 22 avril 2022. URL : http://journals.openedition.org/ahrf/8393 ; DOI : https://doi.org/

10.4000/ahrf.8393

Ce document a été généré automatiquement le 22 avril 2022.

Tous droits réservés 


\title{
Mœurs, vertu et corruption : Sade et le républicanisme classique
}

\author{
Martin Nadeau
}

Les thèmes des mœurs, de la vertu et de corruption sont cruciaux au sein de ce que les historiens désignent par le paradigme du républicanisme classique. Développés notamment par Machiavel et les républicains du XVII ${ }^{e}$ siècle en Angleterre, ces thèmes se sont avérés omniprésents chez les auteurs qui ont abordé les questions politiques tout au long du siècle suivant, notamment lors des Révolutions américaine et française. Les humanistes civiques de la Renaissance estiment que la corruption du corps politique advient lorsque les citoyens attendent de représentants ce qu'ils devaient attendre d'eux mêmes et d'eux seuls, en qualité de membres de la République ${ }^{1}$. Cette définition politique de la corruption précise un sens initial qui désigne un processus de décrépitude morale où l'intérêt particulier domine l'intérêt général, et s'impose à lui.

2 J'aimerais élucider ici une relation que Sade entretient avec le paradigme du républicanisme classique ou avec ce qu'un de ses contemporains et compatriotes, Benjamin Constant, appelle dans un discours fameux la Liberté des Anciens. Cette dernière repose sur la participation directe des citoyens aux affaires publiques. Elle se distingue de la Liberté dite des Modernes, qui a pour fondement le système représentatif par lequel, comme le dit Constant, « une nation se décharge sur quelques individus de ce qu'elle ne peut ou ne veut pas faire elle-même». La Liberté des Modernes, en effet, doit permettre aux "intérêts privés " d'occuper davantage de temps. Constant a pu toutefois très bien reconnaître qu'être «absorbé dans la jouissance de notre indépendance privée » constitue le «danger» de cette forme de liberté ${ }^{2}$.

3 Je me confronte d'emblée ici à une double querelle historiographique, tant à l'égard de la figure controversée du marquis de Sade, que du rôle, voire de l'existence même, du paradigme du républicanisme classique au sein de la culture politique occidentale. D'une part, Sade a droit à une abondante littérature critique partagée entre les écueils de la légende noire et de la légende dorée. D'aucuns parmi les récents exégètes de l'œuvre et de la vie du marquis de Sade - et qui ont su éviter ces deux écueils - ont 
souligné son "opportunisme politique " à propos de son action et de ses écrits, produits, diffusés ou publiés lors de la Révolution française ${ }^{3}$. Sur ce front, je tenterai de mettre en relief une cohérence des écrits de Sade (théâtre, opuscules politiques et romans) qui peut être appréciée à l'aune du républicanisme classique et plus particulièrement à partir des thèmes des mœurs, de la vertu et de la corruption. D'autre part, sur un horizon beaucoup plus vaste, la question du républicanisme classique a fait l'objet ces dernières années d'un mouvement antagoniste de l'historiographie, tendant à la fois à étendre son rayonnement, notamment en appliquant les travaux de John G. A. Pocock à la France du dix-huitième siècle, incluant la période révolutionnaire ${ }^{4}$, et à restreindre son importance en terres anglo-saxonnes, voire à compromettre la pertinence, à toute fin pratique, d'une distinction entre «Républicanisme classique » et «Libéralisme " .

Restituer la cohérence d'un paradigme sans appauvrir les relations qu'il entretient avec des paradigmes concurrents, par des processus d'appropriation, de reformulation et de transformation, n'est pas chose aisée. Quentin Skinner et l'École de Cambridge ont développé une épistémologie et une méthodologie de l'histoire des idées permettant d'éviter d'accorder aux textes d'un auteur un sens que le langage de ce dernier ne pouvait formuler ou même concevoir ${ }^{6}$. À cet égard les "partisans" respectifs du libéralisme ou du républicanisme qui se livrent à des jeux savants de citations, en tentant de situer alternativement la pensée d'auteurs ou d'hommes politiques tantôt dans la sphère de l'homo civicus, tantôt dans celle de l'homo ceconomicus, ne nous avancent guère. Également décevantes, m'apparaissent les velléités de décliner ces paradigmes en forgeant de nouvelles appellations telles "Liberal Republicanism $»^{7}$ ou encore "Aristocratic Liberalism ${ }^{8}$. Il peut être davantage fertile, comme l'a suggéré Quentin Skinner", d'étudier ces paradigmes moins à partir d'un corpus d'auteurs canoniques, et dont l'appartenance à l'un ou l'autre varierait selon les citations choisies et des modalités d'emprunts, de références explicites ou de vague influence, qu'à partir de thématiques précises permettant de cerner les enjeux spécifiques propres à chacun. Trois aspects plus spécifiques, sous l'égide des thèmes généraux des mœurs, de la vertu et de la corruption, retiendront ici l'attention : celui de la corruption inhérente à «la puissance de l'or»; de la corruption liée aux institutions politiques représentatives; celui de la formation d'une armée permanente et soldée en constraste avec la création d'une milice de citoyens armés.

5 En conformité avec la chronologie des œuvres de Sade, j'aborderai dans un premier temps une pièce de théâtre, Le comte Oxtiern ou Les Malheurs du libertinage, jouée à Paris pour la première fois le 22 octobre 1791, avec un modeste succès, sur la scène du Théâtre Molière. Il s'agit de la seule pièce, parmi toutes celles que ce "théâtromane " ait composées, à avoir connu également la forme d'une publication imprimée. Dans un second temps, j'insisterai sur deux opuscules de Sade qui soulèvent frontalement la question de la corruption en préconisant certains mécanismes politiques. Le premier, daté du 1er novembre 1792, concerne le mode de sanction des lois par les citoyens assemblés, et non par une assemblée représentative; le deuxième, daté du 1er juin 1793, a été publié au sujet de la création d'une armée soldée à Paris. Il s'agit en quelque sorte, dans les deux cas, de la question de la corruption inhérente à toute représentation. Je m'attacherai enfin à quelques considérations sur la représentation de la corruption dans l'œuvre romanesque de Sade. 
6 Le marquis de Sade a d'abord épousé la Révolution dans la perspective de l'établissement d'une monarchie constitutionnelle, prémunie contre les abus et le despotisme de l'Ancien Régime ${ }^{10}$. Il adopte des positions républicaines au lendemain de la fuite du roi et de son arrestation à Varennes le 21 juin 1791. Dans son « Adresse d'un citoyen de Paris au roi des Français », lue publiquement et diffusée trois jours plus tard, il se montre encore attaché malgré tout à la personne du monarque, dont la fuite est attribuable, selon lui, à une machination orchestrée par " des courtisans corrompus » et des «prêtres fanatiques $»^{11}$. C'est d'ailleurs un courtisan corrompu que Sade met en scène dans sa pièce Le comte Oxtiern.

7 L'œuvre dramatique de Sade - une vingtaine de pièces écrites pour la plupart lors de ses séjours carcéraux à Vincennes et à la Bastille - a fait l'objet de controverses ${ }^{12}$ qui sont abordées dans l'étude de Sylvie Dangeville remarquant d'emblée que, de l'aveu du principal intéressé, sa production dramatique était celle à laquelle il «tenait le plus ${ }^{13}$. Mais on sait que cette carrière d'auteur dramatique a subi de multiples infortunes. Le comte Oxtiern constitue le seul « succès » véritable de Sade. Le public parisien n'a pu voir cette pièce qu'à deux reprises : à la première du 22 octobre 1791, succéda un seul autre spectacle, le 4 novembre suivant, sur les planches du même théâtre, le Théâtre Molière, spécialisé dans le répertoire patriotique. Le Moniteur universel y consacre tout de même un compte rendu détaillé, où l'on peut lire «Le comte Oxtiern ou les Effets [sic] du libertinage, drame en trois actes, en prose, a été représenté avec succès sur ce théâtre ${ }^{14}$. Le directeur du Théâtre Molière exprima en outre le souhait que d'autres pièces de Sade soient jouées afin que le public puisse être davantage en mesure d'apprécier le talent de l'auteur ${ }^{15}$. Enfin, une dernière représentation d'Oxtiern - avec le titre amputé du mot «Comte »- eut lieu huit ans plus tard, le 13 décembre 1799, sur les planches de la Société Dramatique de Versailles. Sade lui-même y jouait le rôle de Fabrice, l'aubergiste vertueux qui s'oppose aux machinations du sénateur scélérat Oxtiern; et ses talents d'acteurs ont été pareillement reconnus ${ }^{16}$.

8 Le thème de la corruption du sénateur Oxtiern est central dans cette pièce, à l'instar de son œuvre en général, incluant d'autres pièces de théâtre, telles, en 1783, Le Prévaricateur ou encore Le Suborneur - deux termes quelque peu vieillis dans la langue française pour désigner un registre de la corruption au sein des fonctions publiques. Ces pièces se distinguent d'Oxtiern par le refus systématique essuyé auprès de plusieurs administrations de théâtres auxquelles Sade les avait soumises et conséquemment par l'inexistence de représentation publique attestée ${ }^{17}$. Soulignons enfin qu'il n'y a rien d'obscène dans son théâtre, rien de pornographique: comme le dit Maurice Lever: " point de lubricité, point d'hystérie, point d'imprécations, aucun de ces supplices par lesquels Sade exorcise nos consciences ${ }^{18}$. Ce qui explique en partie la perplexité de certains de ses exégètes.

9 L'intrigue en est simple, connue, mais je me permettrai de la résumer succinctement. Oxtiern, sénateur suédois très en vue à la cour de Stockholm, use de son pouvoir, de ses relations et surtout de son or afin d'assouvir le désir tyrannique l'enchaînant à Ernestine, jeune personne de seize ans qui « passait avec juste raison pour l'une des plus belles créatures qu'on eût encore vue en Suède ». Oxtiern doit pour cela écarter l'amant de cette dernière, Herman, ce qui s'exécute sans trop de difficultés grâce au pouvoir dont jouit le sénateur. Toutefois, l'intervention de Fabrice, l'homme vertueux de l'auberge où se déroule l'intrigue, permettra à Herman ainsi qu'au père d'Ernestine de délivrer celle-ci des machinations orchestrées par le sénateur corrompu ; ce dernier 
périt à la fin de deux coups de pistolet infligés par l'amant. Dénouement heureux, contrairement à celui de la nouvelle Ernestine, dont est tirée la pièce, et que Sade avait rédigée antérieurement lors de son emprisonnement à la Bastille. Sade publiera la nouvelle en 1800 dans son recueil Les crimes de l'amour ${ }^{19}$.

La nature de plusieurs répliques de la pièce Le comte Oxtiern permet d'effectuer cette relation entre la représentation de la corruption et la thématique, inhérente au républicanisme classique, des menaces que font peser les richesses mobilières, l'argent, l'or ou le numéraire, sur la vie d'une communauté politique ou d'une République sagement ordonnée. Plus spécifiquement, dans le contexte français de l'automne 1791, les débats sur les problèmes que pose l'agiotage vont bon train; d'autres pièces de théâtre, telle Les Portefeuilles de Collot d'Herbois, mettent en scène cette problématique ${ }^{20}$. Dès la première scène du premier acte de la pièce de Sade le débat est lancé21. Mais les répliques les plus suggestives de l'importance de cette thématique chez Sade sont certainement celles que s'échangent Oxtiern et Derbac - ami et confident du sénateur - à la scène première du second acte, alors que le sénateur médite sur les moyens de se débarrasser de l'amant d'Ernestine, à l'aide de «faux témoins » et de "juges corrompus ", sans se soucier des objections moralisatrices de Derbac s'écriant ultimement: «Et les lois, mon ami, les lois?»; ce à quoi Oxtiern répond laconiquement : « Je ne les ai jamais vu résister à la puissance de l'or ${ }^{22}$.

11 La représentation de la corruption inhérente à la "puissance de l'or", et plus généralement aux hautes fonctions politiques et judiciaires, est cruciale dans cette pièce : elle scande l'ouverture des deux premiers actes et caractérise le développement de toute la pièce. Ceci permet de placer Sade en relation avec plusieurs moralistes français du siècle des Lumières ${ }^{23}$, et peut-être encore plus catégoriquement avec des auteurs anglo-saxons liés au républicanisme classique, parfois méconnus ${ }^{24}$, ou mieux connus tels John Trenchard et Thomas Gordon, dans leurs Cato's Letters ${ }^{25}$; ou encore John Brown, The Estimate of the Manners and Principles of the Time (London, 1757) ${ }^{26}$. Tous auteurs chez qui les concepts de mœurs (manners), de vertu (virtue) et de corruption présentaient de vives préoccupations, dans un contexte de "changements économiques dévastateurs ${ }^{27}$.

12 L'auteur du roman Les Infortunes de la vertu ${ }^{28}$ a également été pamphlétaire. Témoin des journées révolutionnaires d'août 1792 ainsi que des massacres de septembre ${ }^{29}$, il n'exprime aucun regret, nonobstant ses positions antérieures à l'égard du modèle de la monarchie constitutionnelle, et s'engage ouvertement pour la République. Son activité a pour cadre la section des Piques - qui est aussi, je le rappelle, la section de Robespierre -, où il occupe diverses fonctions administratives. C'est devant l'assemblée générale de cette section, le 1er novembre 1792, que Sade prononce un discours sur le mode de sanction des lois ; la section en vote aussitôt l'impression et la diffusion sous la forme d'un pamphlet intitulé Idées sur le mode de la sanction des lois.

La rhétorique du ci-devant amorce ainsi l'opuscule : «Citoyens, la plus importante de toutes les questions s'élève, et rien n'est singulier comme la lenteur que vous paraissez mettre à y répondre : je gémis des motifs qui tiennent vos idées captives, et j'avoue que je ne les conçois pas $»^{30}$. La question de la sanction des lois est en effet fondamentale pour l'élaboration d'une constitution dite républicaine, opérant le transfert de souveraineté du roi au corps de la Nation. En partant du principe que la souveraineté de la Nation est une, indivisible et inaliénable, Sade estime que les députés ou les représentants à qui ont été délégués momentanément une portion de cette 
souveraineté afin de rédiger une constitution, n'ont d'autres droits que celui de soumettre des idées au peuple. À ce dernier, seul, appartient le refus ou l'acceptation, c'est-à-dire la sanction, de ces idées. Afin de bien établir ces premiers principes, il a recours à une métaphore que l'on retrouve fréquemment chez les auteurs anglais du XVIII ${ }^{e}$ siècle dont j'ai évoqué les noms plus haut, et notamment chez James Burgh, dont les Political Disquisitions, publiés en 1775, ont constitué une véritable "encyclopédie » politique de la Révolution américaine ${ }^{31}:$ «[L]e pouvoir, en un mot, de vos mandataires est comme le rayon du soleil réfléchi par le verre ardent; vous êtes le faisceau de lumière que je compare à l'astre du jour; vos députés sont le verre brûlant, qui ne possèdent que ce qu'ils ont reçu de vous, et qui n'éclaireront la terre que des feux que vous leur aurez transmis ${ }^{32}$.

Pour Sade, il s'agit de prévenir l'abus du pouvoir délégué. L'opuscule Idées sur le mode de la sanction des lois affirme que le peuple ne transmet qu'une portion de sa souveraineté : celle de proposer des lois. Le peuple, en fait, conserve pour lui une part importante de sa souveraineté sous la forme de la sanction des lois, c'est-à-dire le pouvoir de choisir parmi les lois proposées; de les refuser ou de les accepter. "Tout le mystère d'une république, soutenait James Harrington, l'une, sinon la principale source du républicanisme classique anglais, ne consiste qu'à faire les parts ou qu'à choisir $»^{33}$. La république idéale définie par cet auteur dans The Commonwealth of Oceana est un régime mixte constitué d'une assemblée composée du petit nombre (correspondant au pouvoir aristocratique), qui propose, et d'une assemblée formée du grand nombre (pouvoir démocratique), qui dispose.

15 En s'interrogeant sur la nature de cette seconde assemblée chargée de choisir ou de sanctionner les lois, Sade rejette l'idée que cette assemblée sanctionnante devrait être représentative, c'est-à-dire composée d'un membre par département. Un telle assemblée, dit-il, ne peut-être que la rivale ou l'esclave de la première ; si elle est rivale, alors l'autorité passerait infailliblement dans ses mains et les mêmes vices de la représention " corrompraient très incessamment toutes les opérations des députés " ${ }^{34}$. Si cette assemblée représentative sanctionnante devient au contraire l'esclave de la première, poursuit Sade, alors « les chaînes que vous redoutez auront acquis un degré de pesanteur d'autant plus violent, que deux corps puissants vous les auront imposées ». Il suggère donc plutôt la division de ce pouvoir majeur de la sanction «dans une certaine quantité d'assemblées primaires » qui permet de mieux prononcer les vœux du peuple et d'affaiblir «l'énorme pouvoir de l'énergie sanctionnante " par cette division qui ne lui laisse " que la liberté de faire le bien, sans aucune facilité pour le mal ». Sans cette nouvelle création des assemblées primaires sanctionnantes, dit-il, « autant valait sans doute rester ensevelis sous le fatras informe des lois gothiques de vos ancêtres, et des interprétations plus effrayantes encore des compilateurs qui nous les expliquaient $»^{35}$.

16 Sade décrit ensuite les mécanismes de convocation de ces assemblées primaires permettant de rassembler directement, sans représentation, la France entière. Il repousse l'objection selon laquelle une assemblée de gens éclairés conviendrait mieux qu'une foule bigarrée pour émettre un vœu sur un aussi grave objet en reprenant la thèse du républicanisme classique sur les régimes mixtes, telle que développée par Harrington ; s'il faut des hommes choisis pour proposer des lois - c'est-à-dire une forme d'aristocratie - , c'est le pouvoir démocratique qui doit sanctionner les lois : «C'est le seul vœu du peuple, qui doit approuver ou non les lois faites pour le captiver; il faut 
donc qu'il s'y trouve en masse, sans élection ; l'élection, toujours le résultat du choix, placerait alors pour adopter ou pour rejeter la loi, celui qui malheureusement a le plus souvent l'art de l'éluder, ou le moyen de s'y soustraire, et c'est précisément là l'écueil qu'il faut éviter avec le plus de soin». Une autre métaphore récurrente du républicanisme classique appuie son propos: «Solon disait que les lois étaient comme des toiles d'araignée, à travers lesquelles passaient les grosses mouches, tandis que les petites y restaient seules enveloppées [sic]. Cette comparaison d'un grand homme nous conduit à reconnaître la nécessité d'admettre essentiellement, et peut-être même de préférence, à la sanction d'une loi, cette partie du peuple la plus maltraitée du sort, et puisque c'est elle que la loi frappe le plus souvent, c'est donc à elle à choisir la loi dont elle consent à être frappée ${ }^{36}$.

Cette dernière métaphore n'est pas sans provoquer des résonances avec les thématiques de son œuvre littéraire, à l'instar de la conclusion de cet opuscule où Sade affirme ne pas se méfier de la personne des représentants en tant que telle, mais cherche plutôt à combattre la corruption inhérente à la représentation politique par cette création d'assemblées primaires appelées à sanctionner les lois: "Je ne soupçonne qui que ce soit, je ne me méfie de personne ; aucun individu dans le monde n'a peut-être plus de confiance que moi dans nos représentants, mais je sais jusqu'où va l'abus du pouvoir ; je démèle toutes les ruses du despotisme ; j'ai étudié les hommes et je les connais; je sais qu'ils renoncent avec bien de la peine au pouvoir qui leur est confié, et qu'il n'est rien de difficile comme de poser des bornes à l'autorité déléguée $»^{37}$.

18 Sade publie un autre opuscule politique qui se présente sous la forme d'un «Projet de pétition des sections de Paris à la Convention nationale». Il est daté du 1er juin 1793, soit très exactement durant l'expulsion des députés girondins lors des journées révolutionnaires du 31 mai au 2 juin 1793. Le problème qu'aborde cette pétition est celui de la création d'une « armée soldée de 6000 hommes à Paris » que vient tout juste de décréter la Convention, à l'instigation des adversaires des Girondins, tel que Robespierre $^{38}$. Les arguments que Sade y oppose s'enracinent pleinement dans le terreau du républicanisme classique pour lequel l'établissement d'une armée soldée ou permanente constitue le plus sûr moyen d'attenter à la liberté politique ${ }^{39}$. Il considère que ce décret est politiquement dangereux car « une armée révolutionnaire dans Paris ne seroit qu'une garde prétorienne, dont les ambitieux ou les usurpateurs profiteroient bientôt pour nous donner des fers $\star^{40}$. Le contexte immédiat de la rédaction de cette pétition suggère qu'une telle "garde prétorienne » ait pu être utilisée par Robespierre et ses acolytes pendant ou après l'assaut contre la Convention conduisant à l'expulsion des Girondins. Quoi qu'il en soit, Sade conclut cette pétition par un vœu d'ordre général rappelant ses harangues précédentes où il exhortait les citoyens français, au nom des sacrifices déjà effectués pour la cause de la liberté, à faire encore un effort... - si j'ose anticiper sur le titre du pamphlet lu par Dolmancé dans le texte du roman La Philosophie dans le boudoir ${ }^{41}$ - , plus précisément à se constituer eux-mêmes en milice et non à se reposer sur la représentation d'une armée soldée : «Non, dit-il, jamais les François ne seront assez faibles pour n'avoir fait autant de sacrifices à la liberté, que dans la seule vue de se replonger dans l'esclavage ; et le même serment que nous nous faisons ici, de ne jamais souffrir d'armée soldée dans notre sein, nous l'employons pour nous jurer que c'est avec notre corps que nous formerons le rempart qui nous défendra des ennemis du dehors, ou des traîtres de l'intérieur, souvent les plus dangereux $»^{42}$. 
19 Depuis l'époque de l'Assemblée nationale constituante, où il siègeait comme député d'Arras, l'épithète d' " Incorruptible », comme on le sait, s'est attachée à Robespierre. Ce dernier a su incarner cette volonté implacable de démasquer les traitres de l'intérieur, de déjouer leurs machinations et conspirations, réelles ou supposées, afin de régénérer le peuple français en éradiquant toute forme de corruption. L'instrument de cette régénération est la politique dite de la Terreur, qui s'institutionnalise à l'été 1793 . Que l'Incorruptible lui-même en tant que membre de la section des Piques ait pu fréquenter quotidiennement l'auteur de La Philosophie dans le boudoir a sans doute d'abord inspiré à Sade la nécessité d'exprimer son patriotisme avec emphase. Une occasion lui est offerte après l'assassinat de Marat par Charlotte Corday, qui inspire son célèbre discours prononcé à la fête décernée par la section des Piques aux mânes de Marat et de Le Pelletier, le 29 septembre 1793. Des références à Scévole et à Brutus amorcent le récit de la machination qui a conduit à l'assassinat de Marat - il évoque "les poignards de Machiavel » - , tandis que le portrait de la conspiratrice Charlotte Corday est tracé d'un pinceau résolument sadien : "Sexe timide et doux, comment se peut-il que vos mains délicates aient saisi le poignard que la séduction aiguisait ? [...] Le barbare assassin de Marat, semblable à ces être mixtes auxquels on ne peut assigner aucun sexe, vomi par les Enfers pour le désespoire de tous deux, n'appartient directement à aucun $»^{43}$.

En dépit des efforts de patriotisme qu'il a déployés, Sade reprend le chemin des prisons en décembre 1793, et ce pour plusieurs raisons. Son "modérantisme", tout d'abord, était en cause. J'aimerais insister ici sur le fait que le «modérantisme» de Sade en pleine Terreur, à l'instar de "l'indulgence » de Camille Desmoulins, constitue une prise de position politique à la fois précise et ferme, que l'on ne saurait en aucun cas taxer d'opportuniste ; je rappelle que Desmoulins a été guillotiné, avec les « Dantonistes » ou "Indulgents ", le 6 avril 1794, pour avoir défendu cette même option politique. À maintes reprises les fonctions de Sade à la section des Piques lui ont permis de se prononcer contre des formes de violence ou de délation, contre la peine de mort, en plus de défendre certaines victimes du soupçon et de la méfiance exacerbée, tel l'ancien ministre de l'Intérieur Roland de la Platière ${ }^{44}$. Le fait qu'il soit un ci-devant marquis, père de deux émigrés, et surtout le fait que son nom ait figuré par erreur sur une liste de nobles émigrés ont certainement attisé les soupçons à son égard. Les positions classiques qu'il a défendues contre le décret de la Convention sur la formation d'une armée soldée ont pu jouer également un rôle. Parallèlement, un document issu d'une maison d'arrêt où Sade a effectué un bref séjour, en tentant de cerner le caractère et les opinions du ci-devant, dénonce le fait qu'il avançait «continuellement dans ses conversations particulières des comparaisons tirées de l'histoire grecque et romaine pour prouver l'impossibilité d'établir un gouvernement démocratique et républicain en France $»^{45}$. Une des dernières tâches de Sade à la section des Piques avant son arrestation a été d'ailleurs de présider une commission de toponymie chargée de «changer le nom des rues de son arrondissement qui portent des inscriptions proscrites, ignobles ou insignifiantes ", et de les remplacer par ceux de Lycurgue, Caton, Solon, Spartacus... ${ }^{46}$.

21 Alors que Sade subit sa "détention nationale » et que s'emballent les engrenages de la Terreur, d'autres républicains ont pu déjà mettre en cause cette dynamique de la Révolution : «[...] il semble que la révolution n'ait d'autre but que de scruter la moralité des 25 millions d'individus qui composent la république ", écrit Jean-Baptiste Salaville dans Les Annales patriotiques et littéraires du 17 février $1794^{47}$. Il suggère que cette volonté 
absolue de régénération pourrait constituer en fait une forme absolue de corruption: « [L]a conspiration la plus dangereuse seroit peut-être celle qui tendroit à corrompre l'esprit public, en généralisant le soupçon, la méfiance et la terreur $»^{48}$.

Sauvé de justesse par la réaction thermidorienne, et aussi par le désordre administratif engendré par l'encombrement des prisons, Sade se remet activement à écrire et met en question à sa manière le problème de cette corruption qu'a éprouvé la première république française sous le régime de l'Incorruptible. Il écrit aux législateurs de la Convention en octobre 1794 afin de demander le rapport total de la loi du 17 septembre 1793, dite loi des suspects, l'une des bases juridiques de la Terreur, dont les «abus énormes » n'ont servi qu'à " paralyser les arts, le commerce, l'agriculture, qu'à semer le trouble, la division dans les familles, qu'à faire négliger l'éducation des enfants, qu'à produire en un mot les plus funestes effets, en arrêtant les deux tiers de la France ${ }^{49}$. Le mois suivant, il décrit à son avocat Gaufridy sa propre expérience de la Terreur dans une lettre, dont les termes - et la macabre statistique - ne dépareilleraient pas ses romans les plus "poivrés »: «Picpus, enfin, était un paradis terrestre; belle maison, superbe jardin, société choisie, d'aimables femmes; lorsque tout à coup la place des exécutions s'est mise positivement sous nos fenêtres et le cimetière des guillotinés dans le beau milieu de notre jardin, nous en avons mon cher ami enterré 1800 en 35 jours, dont un tiers de notre malheureuse maison; enfin mon nom venait d'être mis sur la liste et j'y passais le 11, lorsque le glaive de la justice s'est apesanti la veille sur le nouveau Sylla de la France $»^{50}$.

Quoique le Comité de Sûreté générale ait autorisé Sade à rester à Paris afin de continuer «à mouvoir l'esprit public par [s]es ouvrages patriotiques " ${ }^{51}$, sa plume s'exerça désormais sur d'autres registres. Avec son roman La Philosophie dans le boudoir, publié en 1795 , il aborde frontalement la question des mœurs dans ses rapports avec la politique. Il s'agit naturellement d'un thème important du républicanisme classique, omniprésent dans la littérature du dix-huitième siècle en France, chez Helvétius, Mably, Montesquieu, Rousseau, parmi plusieurs autres philosophes liés de près ou de loin à ce paradigme. Ce n'est que par l'excercice des vertus domestiques qu'un peuple apprend la pratique des vertus politiques, écrit Mably dans ses Entretiens de Phocion (1763) mettant en scène un disciple de Platon ${ }^{52}$. Dans La Philosophie dans le boudoir, Sade reprend cette formule $\mathrm{du}$ dialogue platonicien ${ }^{53}$; mais avec comme sous-titre "Les instituteurs immoraux ", s'annonce d'emblée une perspective détournée des rapports platoniciens entre mœurs et politique, et peut-être même une véritable satire ou dérision du discours jacobin sur la nature et la vertu. L'idée de camper des dissertations de philosophie politique, dont la lecture du célèbre opuscule « Français, encore un effort si vous voulez être républicains", au sein d'un boudoir - lieu par excellence de la jouissance de l'indépendance privée - où trois libertins aguerris réussissent à corrompre une jeune personne - Eugénie - qui s'exclame à la fin : «votre ouvrage est fini ; ce que les sots appellent la corruption est maintenant assez établie dans moi pour ne laisser aucun espoir de retour ", pose une question éminemment politique ${ }^{54}$. Les propos du principal instigateur de cette corruption, Dolmancé, déclarant «Que les lois que nous promulguons n'aient pour but que la tranquillité du citoyen, son bonheur $»^{55}$, confiant que "pourvu que je sois heureux, le reste m'est absolument égal ${ }^{56}$ et concluant enfin: "Voilà une bonne journée! Je ne mange jamais mieux, je ne dors jamais plus en paix que quand je me suis suffisamment souillé dans le jour de ce que les sots appellent des crimes $»^{57}$, peuvent interpeller les critiques de la Liberté des 
Modernes soupçonnant l'espèce de despotisme que le repli des hommes sur leurs intérêts privés est de nature à engendrer ${ }^{58}$.

Dans son roman La Nouvelle Justine, suivie de l'Histoire de Juliette sa sœur publié entre 1797 et 1803 , Sade dessine le personnage Saint-Fond, ministre et courtisan. Celui-ci constitue sans doute l'archétype de l'homme politique scélérat et corrompu. Un peu selon la disposition de Dolmancé avec Eugénie, Saint-Fond prodigue à Juliette des leçons de politique tirées directement des historiens latins comme Salluste, auxquels les adversaires du régime de Walpole en Angleterre, tel que Thomas Gordon, se référaient régulièrement afin de mieux faire sentir les effets de ce qu'ils nommaient la corruption généralisée : «Apprends, Juliette, qu'il est de la politique de tous ceux qui mènent un gouvernement d'entretenir dans les citoyens le plus extrême degré de corruption; tant que le sujet se gangrène et s'affaiblit dans les délices de la débauche, il ne sent pas le poids de ses fers; on peut l'en accabler sans qu'il s'en doute. La véritable politique d'un État est donc de centupler tous les moyens possibles de la corruption du sujet $\|^{59}$.

Il ne s'agit pas d'interpréter les romans de Sade mettant en scène de telle figure de corruption en tant que programme préconisant tel ou tel système. Parmi les nombreuses notes qui ponctuent ses ouvrages, et par lesquelles l'auteur entretient une relation de complicité avec ses lecteurs, en anticipant ou en provoquant ses réactions, se trouve un avertissement à ce sujet dans le roman épistolaire Aline et Valcour (1793-1795). S'adressant à Léonore, après avoir échappé aux griffes du corrégidor de Lisbonne, Clémentine lui explique qu'il faudrait que le magistrat n'ait l'autorité « qu'un instant, et que la crainte d'être traité dans l'état faible, comme il traitait les autres quand il dominait, servît toujours de frein à ses passions ». Sade aussitôt apostrophe le lecteur : "Quelques lecteurs vont dire: Voilà une bonne contradiction; on a écrit quelque part avant ceci qu'il ne fallait pas changer souvent les ministres de place : ici l'on dit tout le contraire. Mais ces vétilleux lecteurs veulent-ils bien nous permettre de leur faire observer que ce recueil épistolaire n'est point un traité de morale dont toutes les parties doivent se correspondre et se lier ». Il suggère donc : « Au lieu de s'attacher à démêler des contradictions ou des redites, choses inévitables dans une pareille collection, il faut que le lecteur, plus sage, s'amuse ou s'occupe des différents systèmes présentés pour ou contre, et qu'il adopte ceux qui favorisent le mieux, ou ses idées, ou ses penchants ${ }^{60}$. Ainsi, ce n'est pas seulement par ses tableaux de la corruption que l'œuvre romanesque de Sade peut entretenir des correspondances avec le républicanisme classique. Cette cohérence peut être appréciée en rapport avec ses opuscules et son action politiques suscitant la résistance du citoyen face aux formes passives de la représentation.

Comme second point de conclusion, j'aimerais proposer deux pistes susceptibles d'offrir de nouvelles lumières quant à la position et à la cohérence politique du marquis de Sade, dont il ne s'agit pas de donner le fin mot. La première, est suggérée par André J. Bourde dans "Sade, Aix et Marseille : Un autre Sade », qui met en relief le fait que la condamnation de Sade lors de l'« affaire de Marseille » en $1772^{61}$ ait été prononcée par les " parlements Maupeou ». Ce qui peut amener à situer cette condamnation dans une perspective politique, c'est-à-dire celle de la polémique opposant une ancienne noblesse de souche ${ }^{62}$ et d'anciens parlementaires rassemblés contre les nouveaux nommés par le chancelier Maupeou en février $1771^{63}$.

Une seconde piste réside dans la poursuite de l'analyse du roman Aline et Valcour, une œuvre à laquelle Sade se montrait particulièrement attaché, à l'instar de son théâtre, et 
qui se distingue également par l'absence de toutes représentations obscènes ou pornographiques. Jean-Marie Goulemot, dans «Lecture politique d'Aline et Valcour. Remarques sur la signification politique des structures romanesques et des personnages ", s'attarde sur le problème que posent de multiples passages de ce roman quant à leurs éventuels accents prophétiques au sujet de la Révolution française; comme il le remarque : "On peut lui [Sade] rétorquer qu'il était aisé de prédire dans un livre composé en 1788 , mais publié en 1793 , la révolution de $1789 »^{64}$. Quoi qu'il en soit, parmi ces passages en question, plusieurs mettent en jeux des thématiques inhérentes au républicanisme classique, notamment dans sa dimension critique à l'endroit des richesses capitalistes et des "monied interests». Je me permettrai d'en citer un seul passage, peut-être long; il sort de la bouche du curé de Berseuil déclarant à Déterville :

«On ne veut point voir de pauvres en France; eh bien! que l'on s'occupe, pour y réussir, du moyen de réformer les mœurs, et de préserver surtout la jeunesse de leur perfide corruption; que l'on réforme le luxe, ce luxe pernicieux qui ruine et dérange le riche sans soulager le misérable, et qui plonge bientôt celui-ci dans l'abîme, par sa folle prétention à atteindre ce qu'il ne peut approcher qu'en entraînant sa perte. [...] Diminuez les impôts, honorez, encouragez l'agriculture, préférez surtout l'honnête individu qui s'y livre à cet impertinent plumitif qui, masqué d'une jupe noire, a quitté la charrue de son père, pour venir s'engraisser, dans la ville, des divisions intestines du citoyen. Classe abjecte, venimeuse, aussi inutile que méprisable, que de bonnes lois devraient ou retenir dans ses foyers, ou enchaîner, dès qu'elle en sort, à des travaux publics, dans lesquels, plus utiles au moins, ou qu'au parquet ou qu'au barreau, elle servirait la patrie, au lieu de la détruire, au lieu de la miner sourdement par ses prévarications, ses rapines et ses escroqueries scandaleuses. [...] Votre Babylone moderne s'anéantira comme celle de Sémiramis, elle s'évanouira de dessus le globe de la terre, comme ont disparu ces villes florissantes de la Grèce, qui n'ont eu comme elle que le luxe pour cause de leur dépérissement; et l'État énervé pour embellir cette nouvelle Sodome, s'engloutira comme elle, sous ses ruines dorées*. [Note de Sade] * C'est ici, comme dans bien d'autres passages, que nous supplions nos lecteurs de ne pas perdre de vue que cet ouvrage s'écrivait un an avant la Révolution ${ }^{65}$ ».

Enfin, puisque je me refuse ici à donner le dernier mot sur les positions politiques de Sade - aristocrate républicain ? démocrate aristocratique ? - acteur politique et auteur complexe assurément ${ }^{66}$, tout comme Machiavel avec lequel il partage d'ailleurs l'insigne honneur pour un homme de lettres d'avoir donné, par son nom même, aux idiomes français et anglais un substantif d'usage courant, je me contenterai d'effectuer en terminant ce clin d'œil à Rousseau : "Je n'assiste jamais à la représentation d'une comédie de Molière que je n'admire la délicatesse des spectateurs. Un mot un peu libre, une expression plutôt grossière qu'obscène, tout blesse leurs chastes oreilles; et je ne doute nullement que les plus corrompus ne soient toujours les plus scandalisés ${ }^{67}$.

\section{NOTES}

1.John G.A. Pocock, The Machiavellian Moment. Florentine Political Thought and the Atlantic Republican Tradition, Princeton, Princeton University Press, 1975, rééd. 2003, 634 p., voir 
p. 203-204. Traduction française Paris, PUF, 1998, coll. « Léviathan », avec une préface de Jean-Fabien Spitz.

2.Benjamin CONSTANT, De la Liberté des Anciens comparée à celle des Modernes. Discours prononcé à l'Athénée royal de Paris en 1819, Marcel GAUCHET (éd.), Paris, Hachette, 1980, p. 512.

3.On peut consulter dans l'étude de Laurence L. BongIE, Sade : A Biographical Essay, Chicago, Chicago University Press, 1998, 336 p., une bibliographie critique réunissant les principaux travaux français et anglo-saxons, "Sadean Criticism », p. 281-300, qui est utile, en dépit de l'hostilité affichée de l'auteur envers son sujet, incluant l'accusation d'opportunisme politique (p. 298). Une étude beaucoup moins hostile, celle de Raymond JeAN, Un Portrait de Sade, Arles, Actes Sud, 1989, 330 p., ne parle pas moins, au sujet de la correspondance que le marquis de Sade entretient avec son avocat Gaufridy en 1790, « d'un certain opportunisme qui sera le sien dans la période qui va suivre » (p. 252).

4.Voir Keith Michael BAKER, « Transformations of Classical Republicanism in Eighteenth-Century France », Journal of Modern History, n 73, 2001, p. 32-53 ; Rachel HAMmersley, « Camille Desmoulins's Le Vieux Cordelier : A Link between English and French Republicanism », History of European Ideas, n² 27, 2001, p. 115-32 ; Andrew JAINCHILL, "The Constitution of the Year III and the Persistence of Classical Republicanism », French Historical Studies, vol. 26, n 3, 2003, p. 399-435 ; Raymonde MONNIER, « Républicanisme et Révolution française », French Historical Studies, vol. 26, n 1, 2003, p. 87-118 ; Martin NADEAU, « Machiavel et la première république française ", Carrefour, $\mathrm{n}^{\circ}$ 24, 2002, p. 117-133 ; Johnson Kent WRIGHT, A Classical Republican in Eighteenth-Century France: The Political Thoughts of Mably, Stanford, Stanford University Press, 1997, 261p.

5.Voir parmi ces travaux à travers lesquels tendent à s'estomper les distinctions entre Républicanisme classique et Libéralisme, le recueil de Joyce APPLEBY, Liberalism and Republicanism in Historical Imagination, Cambridge (MA), Harvard University Press, 1992, 351 p. ; Steve PINCUS « Neither Machiavellian Moment nor Possessive Individualism : Commercial Society and the Defenders of the English Commonwealth ", American Historical Review, vol. 103, n 3, 1998, p. 705-736 ; Paul A. RAHE, Republics Ancient and Modern. Classical Republicanism and the American Revolution, Chapel Hill, University of North Carolina Press, 1992, 1201 p.

6.Voir notamment Quentin SKINNER, « Meaning and Understanding in the History of Ideas », History and Theory, vol. 8, 1969, p. 3-53.

7.Vickie B. Sullivan, Machiavelli, Hobbes, and the Formation of a Liberal Republicanism in England, Cambridge (MA), Cambridge University Press, 2004, 284 p.

8.Alan S. KAHAN, Aristocratic Liberalism. The Social and Political Thought of Jacob Burckhardt, John Stuart Mill and Alexis de Tocqueville, New York, Oxford University Press, 1992, 228 p. 9.Quentin Skinner, dans La Liberté avant le Libéralisme, Paris, Liber, 2001, p. 62, exprime ainsi cette recommandation : "Les historiens des idées feraient bien de ne pas s'attacher exclusivement ou même principalement à un canon de textes prétendument classique, mais plutôt à la place qu'occupent ces textes au sein de traditions et de contextes de pensée plus larges ".

10.Voir sur les positions politiques de Sade avant la Révolution, Michel VovelLE, «Sade, Seigneur de village ", dans Le Marquis de Sade, Actes du colloque d'Aix-en-Provence 19-20 février 1966, Paris, Armand Colin, 1968, 308 p., p. 23-41. 
11.Alice M. LABORDE, Correspondance du Marquis de Sade, vol. XXII, Genève, Slatkine Reprints, 1996, p. 53-54. « Adresse d'un citoyen de Paris au roi des français ». 12.Voir la préface de John FRANCESCHINA et Ben OHMART (éds et traducteurs), The Plays of the Marquis de Sade, Durango, Colorado, 1993, volume 1, contrastant l'opinion de deux des plus grands spécialistes de Sade, Gilbert Lely, jugeant négativement cette partie de sa production littéraire, et Annie Le Brun, l'estimant avec plus de faveur.

13.Sylvie DANGEVILLE, Le Théâtre change et représente. Lecture critique des œuvres dramatiques du Marquis de Sade, préface de Maurice LEVER, Paris, Honoré Champion, 1999, p. 17, citant les propos de J-J Brochier, éditeur du Théâtre de Sade (1970) : « Les rapports de Sade avec le théâtre ont été constants pendant toute sa vie, et c'est à son théâtre que, de toute évidence, Sade tenait le plus ».

14. La Gazette Nationale ou Le Moniteur Universel, $\mathrm{n}^{\circ} 310$, dimanche 6 novembre 1791, $\mathrm{p}$. 1293-1294. Ce compte rendu relate certaines turbulences au sein de l'auditoire qui ont provoqué « une espèce de scission dans l'assemblée. Une très faible minorité a fait entendre de timides coups de sifflet, dont l'auteur a été bien dédommagé par les applaudissements nombreux de la majorité. On l'a demandé après la représentation, c'est M. de Sade ".

15. Cité par Franceschina et Ohmart, préface de The Plays of the Marquis de Sade, p. 5. 16.Ibid., p. 6.

17.Voir la notice de cette pièce traduite par Franceschina et Ohmart sous le titre The Shyster or the Magistrate of Long Ago, p. 189-192.

18. Maurice LEVER, préface à Sylvie DANGEVILLE, Le Théâtre change et représente, op.cit, p. 12. 19.SADE, Ernestine. Nouvelle Suédoise, Paris, Gallimard, 2003. Dans cette nouvelle, le dénouement s'avère en effet plus complexe, plus « sadique » peut-être. Herman n'est pas seulement arrêté et emprisonné par Oxtiern, mais exécuté sous les yeux mêmes d'Ernestine, qui défaille, permettant ainsi au sénateur de la violer. Enfin, les machinations du sénateur conduisent également à la méprise du père d'Ernestine qui tuera sa propre fille lors d'un duel qui devait l'opposer à Oxtiern. Ce dernier est toutefois arrêté par la justice et condamné « au bannissement perpétuel dans les mines ». Le père d'Ernestine tente d'obtenir sa libération afin de le provoquer à nouveau en duel ; le comte refuse de se défendre, l'honneur du père interdisant de s'en prendre à un homme désarmé, Oxtiern tente en vain de se suicider puis, grâce au pardon ultime du père, reprend le chemin de la vertu.

20.Voir sur ce point Michel BIARD, Collot d'Herbois : Légendes noires et Révolution, Lyon, Presses Universitaires de Lyon, 1995, 225 p.

21.Je me réfère à Jacques TRUCHET (éd.), Théâtre $d u$ XVIII ${ }^{e}$ siècle, Paris, Gallimard, 1974, vol. II, SADE, Oxtiern, Acte premier, scène première, p. 1084. « Fabrice : Je connais le comte Oxtiern depuis longtemps; et c'est à cause de cela que je parie qu'il n'y a pas un mortel plus dangereux dans toutes les provinces de Suède. / Casimir [valet de chambre du sénateur : Oui ; mais il paie bien. / Fabrice : Et c'est ce qui le rend plus redoutable : il n'y a rien de si pernicieux que l'or dans la main des méchants... Qui peut résister à celui qui possède le plus sûr moyen de toutes les corruptions ?... Mon ami, je voudrais qu'il n'y eût de riches que les honnêtes gens [...].

22.Ibid., Acte second, scène première, p. 1092-93.

23. Voir à ce sujet le livre de Cyril LE MEUR, Les Moralistes français et la politique à la fin du XVIII siècle, Paris, Honoré Champion, 2002, 615 p. 
24. Mentionnons ici Andrew Brown, l'auteur de The Character of the True Publick Spirit Especially with Relation to the ill Condition of a Nation, thro' the Prevalency of the Privat Spirit, Selfish and Sinister Designs (Edinburgh, 1702) ; John DENNIS, An Essay Upon Publick Spirit, being a Satyr in Prose upon the Manners and Luxury of the Times, the Chief Sources of our Present Parties and Divisions (London, 1711); Thomas BASTON, Thoughts on Trade and a Publick Spirit Considered under the Following Heads Viz. I. Companies in Trade II. Stock-jobbers III. Projectors IV. Corruptions in the Law and Public Offices. V. Of a Public Spirit (London, 1716).

25.Voir en particulier Cato's Letters, $\mathrm{n}^{\circ}$ 26, Saturday April 22, 1721 (Indianapolis, 1995) «The sad Effects of general corruption, quoted from Algernon Sidney, Esq ». Cf. p. 190 : «By this means virtue itself becomes popular, as in Sparta, Rome, and other places, where riches (which, with the vanity that follows them, and the honours men give to them, are the root of all evil) [je souligne] were either totally banished, or little regarded $»$.

26.Outre par ces huit éditions consécutives en Grande-Bretagne (1757-1758), cet ouvrage se distingue par l'importance de sa diffusion en France, traduit par Charles Chais, à travers au moins deux éditions contemporaines. Voir WRIGHT, A Classical Republican in Eighteenth-Century France, p. 183 : " Any reader who doubts the power of education and religion to determine the fate of a republic, Mably suggests, should consult the famous book by the British moralist John Brown, Estimate of the Manners and Principles of the Times - ' I know of no more profound work on politics ' Mably insists - a book that accurately predicted the current decadence of British political character ». 27.Voir Pocock, The Machiavellian Moment, op. cit., p. 391 : « The objection to market profit as the basis of civic personality was its mobility ; 'lightly come', said Harrington, 'lightly go'. What a man had he might lose ; what he was, he might lose at the same instant. The superior man, observed Confucius, was not a utensil ; and civic personality was not a commodity ». Cf. p. 422 pour la formule « an era of devastating economic change ".

28.Première version de 1787-1788, dont l'héroïne était Sophie ; seconde version parue en 1791, Justine ou Les Malheurs de la vertu ; troisième version, publiée en 1797, La Nouvelle Justine suivie de l'Histoire de Juliette.

29.LABORDE, Correspondance du Marquis de Sade, vol. XXII, p. 204. Le Marquis à M. Gaufridy, Paris, ce 6 septembre 1792. «Dix mille prisonniers ont péri dans la journée du trois septembre. Rien n'égale l'horreur des massacres qui se sont commis, mais ils étaient justes. La [ci-devant] princesse de Lamballe a été du nombre des victimes ; sa tête portée sur une pique a été offerte aux yeux du roi et de la reine et son malheureux corps traîné huit heures dans les rues après avoir été souillé, dit-on, de toutes les infâmies et de la plus féroce débauche ; tous les prêtres réfractaires égorgés dans les églises où on les tenait renfermés, parmi eux l'archevêque d'Arles, le plus vertueux et le plus respectable des hommes ».

30.SADE, Idées sur le mode de la sanction des lois, Paris, Gallimard, 2003, p. 37.

31.Voir Lance BANNING, The Jeffersonian Persuasion, Ithaca, Cornell University Press, 1978, 305 p., p. 60. Burgh's « work was little less than an encyclopedia of opposition thought » et Gordon S. WooD, Representation in the American Revolution, Charlottesville, 1969, p. 39. "The people's power, declared Samuel Chase, in an image borrowed from James Burgh, ' is like the light of the sun, native, original, inherent, and unlimited by human authority. Power in the rulers or governors of the people is like the reflected light of the moon, and is only borrowed, delegated and limited by the grant of the people' ". 
32.SADE, Idées sur le mode de la sanction des lois, op.cit. p. 39.

33.Il s'agit ici d'une autre célèbre métaphore développée cette fois par James HARRINGTON, The Commonwealth of Oceana (1656), trad. française de P.F. HENRY (1795), Paris, 1995, p. 244-245. «Le moyen d'assurer en cela l'égalité est facile ; il est connu même des petites filles, ce moyen n'étant que ce qu'elles pratiquent en divers cas. On a donné à deux petites filles un gâteau à partager entre elles : afin donc que chacune puisse avoir ce qui lui revient, l'une dit à l'autre : 'Partagez et je choisirai, ou je partagerai et vous choisirez'. Ce point une fois convenu entre elles, c'en est assez : car si celle qui partage le fait inégalement, elle y perd, en ce que l'autre prend la meilleure part ; c'est pourquoi, elle divise également, et elles ont toutes deux ce qui leur appartient ».

34.SADE, Idées sur le mode de la sanction des lois, op.cit. p. 44.

35.Ibid., p. 45.

36.Ibid., p. 48. Voir James BURGH, Political Disquisitions, London, 1774-1775, vol. II, livre 1, chap. I, p. 34 : « To the same purpose was the saying of lord Coke, that no subject, however potent, or subtile, ever jostled with the law, but it broke his neck. But, in our times, it may be said, as Remus remarked to his brother Romulus, 'Laws serve only as cobwebs, to catch the small flies : the great ones break through them ' ".

37.SADE, Idées sur le mode de la sanction des lois, op.cit. p. 49.

38. Voir à ce sujet les développements de Richard COBB, Les Armées révolutionnaires : Instruments de la Terreur dans les départements, Paris, Mouton, 1961-1963.

39.Voir par exemple Thomas GORDON et John TRENCHARD, Cato's Letters, ${ }^{\circ}$ 94, September 15, 1722, «Against Standing Armies », p. 670 : « I have lately met with some creatures and tools of power, who speak the same language now : they tell us that matters are come that pass, that we must either receive the Pretender, or keep him out with bribes and standing armies ; that the nation is so corrupt, that there is no governing it by other means ; and, in short, that we must submit to this great evil, to prevent a greater : as if any mischief could be more terrible than the highest and most terrible of all mischiefs, universal corruption and a military government ».

40. Marquis de SADE, Lettres, Écrits politiques. Présentation de Gilbert LELY, Genève, Slatkine, 1976, p. 226.

41.Voir la très fine analyse que propose Claude LEFORT, "Sade : le boudoir et la cité ", dans Écrire à l'épreuve du politique, Paris, Calmann Lévy, 1992, 395 p., p. 91-111

42.Marquis de SADE, Lettres, Écrits politiques, p. 228.

43.SADE, Euvres complètes, tome III, opuscules politiques, p. 358. Voir aussi Martin NADEAU, « Sade lecteur de Machiavel », Montréal, Carnets d'histoire, 1999, p. 49-53.

44.Voir Raymond JeAn, Un Portrait de Sade, op. cit, p. 263.

45.Alice LABORDE, Correspondance du Marquis de Sade, vol. XXIII, Sade sous la Terreur, «Copie du Tableau de Sade. Paris, Maison d'arrêt du citoyen Coignard, 18 août 1794 », p. 199.

46.Ibid., p. 110. « Projet tendant à changer le nom des rues de l'arrondissement de la section des Piques, 7 novembre 1793 ». À propos de la rue Neuve-des-Mathurins où il habite, Sade écrit qu'elle « se nommera désormais rue Caton. Cet homme célèbre porta l'amour de la patrie jusqu'à l'enthousiasme. À quatorze ans, il demanda une épée pour tuer Sylla, l'un des tyrans de Rome ; il fut l'un des plus grands ennemis de César ; il avait pris deux partis violents, bien dignes d'un républicain : celui de s'exiler si Pompée 
triomphait, celui de se tuer si c'était César. Ce dernier l'emporta : Caton, ne voulant pas survivre au déshonneur de sa patrie, tint sa parole et s'enfonça son épée dans le cœur ». 47.Voir sur ce quotidien, fondé en octobre 1789 par Louis-Sébastien Mercier et JeanLouis Carra et publié jusqu'en 1797, Stefan LemNY, Jean-Louis Carra (1742-1793) : Parcours d'un révolutionnaire, Paris, l'Harmattan, 2002, 415 p.

48. Annales patriotiques et littéraires, $\mathrm{n}^{\circ}$ 412, lundi 17 février 1794, p. 1843.

49.LABORDE, op. cit. vol. XXIII, « Le Citoyen Sade aux Législateurs de la Convention », p.

218.

50.Ibid., « Le citoyen Sade au citoyen Gaufridy, Paris, ce 19 novembre 1794 », p. 232.

51.Ibid., p. 228. « Le citoyen Sade au citoyen Quinquin Leveuf, Paris, 1er novembre 1794. Ma liberté est suivie d'une faveur très rare. Le Comité de Sûreté générale m'autorise, quoique noble, à rester à Paris pour continuer comme je l'ai fait précédemment à mouvoir l'esprit public par mes ouvrages patriotiques".

52.Voir WRIGHT, A Classical Republican in Eighteenth-Century France, p. 85-86.

53.Comme l'a déjà remarqué Jean-Marie Goulemot dans sa communication intitulée " Beau marquis parlez-nous d'amour ", actes du colloque de Cerisy, Sade, Écrire la crise , Paris, 1983, p. 120.

54.Comme l'exprime Claude LEFORT dans « Sade : le Boudoir et la Cité », op.cit.

55.SADE, La Philosophie dans le Boudoir, Paris, Gallimard, 1976, p. 251.

56.Ibid., p. 258.

57.Ibid., p. 287.

58.Voir par exemple Gordon S. WooD, The Creation of the American Republic, Chapel Hill, University of North Carolina Press, 1969, p. 610. « The problem was, as Charles Thompson lamented in 1786, that most Americans had no other 'Object' than their own individual happiness ".

59.SADE, CEuvres complètes, tome VIII, Juliette ou Les Prospérités du vice, p. 529. Voir en parallèle Thomas GoRDon et John TRENCHARD, Cato's Letters, $n^{\circ} 26$, April 22, 1721, « The sad Effects of general Corruption, quoted from Algernon Sidney », p. 189, et $n^{\circ} 27$ "General Corruption, how ominous to the Publick, and how discouraging to every virtuous Man. With its fatal progress whenever encouraged », p. 194 : «Sallust, or whoever else was the author of the two discourses to Caesar about settling the commonwealth, observes to that emperor, that those magistrates judge wildly, who would derive their own security from the corruption of the people ; and therefore make them wicked men, to make them good subjects ".

60.Aline et Valcour ou le roman philosophique vol. III, lettre XXXVIII, Paris, Jean-Jacques Pauvert, 1963, p. 199, note de Sade.

61.Il s'agit d'une « aventure » de Sade impliquant des prostituées marseillaises, dont les plaintes conduisirent à la « flétrissure publique » du marquis, condamné pour « débauche outrée » par le Parlement d'Aix.

62.La noblesse de la lignée de Sade est attestée depuis au moins le XIII ${ }^{\mathrm{e}}$ siècle. 63.André J. BOURDE, « Sade, Aix et Marseille : Un autre Sade », Le Marquis de Sade, Paris, Armand Colin, 1968, p. 59-70. Voir aussi la retranscription du débat qui a suivi cette communication entre J. Fabre, appuyant cette lecture politique de l'affaire de Marseille, et J. Tulard la contestant et admettant la persécution pour raisons politiques uniquement après 1793.

64. J. M. GOULEMOT, «Aline et Valcour », p. 116. 
65.SADE, Aline et Valcour, vol. I, lettre XVII « Déterville à Valcour », p. 116-118. Goulemot estime avec raison selon moi que ce roman « place Sade plus près de Fénelon, Vauban ou Boulainvilliers et des physiocrates que de Robespierre ou de Saint-Just ». Cf. " Aline et Valcour » p. 117. J. Fabre remarque non moins pertinemment « qu'il n'y a pas du tout de contradiction entre aristocratie et république. En France, au XVIII ${ }^{\mathrm{e}}$ siècle, l'opposition républicaine est d'abord, chronologiquement, une opposition aristocratique ; ce sont les mêmes qui parlent à la fois de retour aux lois fondamentales du royaume et de république ». Ibid., p. 137. Voir aussi à ce sujet Harrold A. ELLIS, Boulainvilliers and the French Monarchy : Aristocratic Politics in Early Eighteenth-Century France, Ithaca, Cornell University Press, 1998.

66.Irène TIEDER, " Marat / Sade. Dramaturgie et scénographie », dans Les Arts de la scène et la Révolution française, sous la direction de Philippe BOURDIN et Gérard LOUBINOUX, Clermont-Ferrand, Presses de l'Université Blaise-Pascal, 2004, p. 545-554, offre une traduction inédite de ce très bel épilogue de Peter Weiss, auteur de la pièce de théâtre dont s'est inspiré Peter Brook : « C'était notre intention dans les dialogues / De présenter des antithèses / Et de les opposer sans cesse / Pour éclaircir les doutes tenaces. / Cependant, de quelque côté que je me tourne / Je ne vois pas de fin à notre drame.../ D'un côté, le désir de changer, d'améliorer le monde / À coups de hache et de couteaux, / De l'autre l'idéologie individualiste, / Qui sombre sous le poids de ses propres pensées. / Ainsi, vous me voyez, dans la situation présente / Toujours devant une question latente ». Cf. p. 554.

67.Jean-Jacques ROUSSEAU, Paris, Garnier-Flammarion, 1971, p. 101, « Dernière réponse de J.-J. Rousseau de Genève ».

\section{RÉSUMÉS}

Cet article examine trois thèmes centraux inhérents au paradigme du républicanisme classique que sont les mœurs, la virtù et le problème de la corruption, à partir de trois formes d'écrits du marquis de Sade: théâtre, opuscule politique et roman. Après un parcours des récentes controverses historiographiques à ce sujet, l'auteur met en relief une cohérence et correspondance entre l'œuvre de Sade et son action politique lors de la Révolution française. D'une part, se distingue l'importance du problème de la corruption liée à la représentation politique ; assemblée représentative en contraste avec les assemblées primaires sanctionnantes, ou encore armée permanente et soldée en opposition à une milice formée de citoyens. De l'autre, se présente le problème de la représentation de la corruption des mœurs, notamment en regard de ce que Sade appelle « la puissance de l'or ». Enfin, cet article réitère la pertinence de l'étude du rayonnement et des transformations encourus par le paradigme du républicanisme classique en sol français.

Mœurs, vertu, and corruption : Sade and classical republicanism. This article examines three central themes inherent in the paradigm of classicial republicanism: múurs, virtu, and the problem of corruption in three types of writings by the marquis de Sade: theater, political pamphlets, and the novel. After a survey of recent historiographical controversies on this subject, the author demonstrates the consistency and connection between the work of Sade and 
his political action at the time of the French Revolution. On one hand is the importance of the problem of corruption linked to political representation; the representative assembly in contrast to the primary assemblies, or a permanent, paid army opposed to a militia formed of citizens. On the other hand, is the problem of the representation of the corruption of múurs, notably in relation to what Sade called, «la puissance de l'or». Finally, this article stresses the importance of a study of the development and transformations of the paradigm of classical republicanism on French soil.

\section{INDEX}

Mots-clés : représentation, mœurs, républicanisme classique, Sade, spectacle, vertu et corruption

\section{AUTEUR}

\section{MARTIN NADEAU}

Faculté des sciences humaines, Université du Québec, Montréal, Canadanadeau.martin@uqam.ca 